Revue internationale P.M.E.

Économie et gestion de la petite et moyenne entreprise

\title{
De l'évaluation des projets de création d'entreprises au pronostic de succès
}

\section{Yves-Frédéric Livian et Stéphane Marion}

Volume 4, numéro 1, 1991

URI : https://id.erudit.org/iderudit/1008027ar

DOI : https://doi.org/10.7202/1008027ar

Aller au sommaire du numéro

Éditeur(s)

Presses de l’Université du Québec

ISSN

0776-5436 (imprimé)

1918-9699 (numérique)

Découvrir la revue

Citer cette note

Livian, Y.-F. \& Marion, S. (1991). De l'évaluation des projets de création

d'entreprises au pronostic de succès. Revue internationale P.M.E., 4(1), 107-129.

https://doi.org/10.7202/1008027ar
Résumé de l'article

On sait la difficulté de dégager des critères d'évaluation de projets de créations d'entreprises ayant une valeur de pronostic.

Cette note présente les résultats d'une étude portant sur un échantillon de 11 cas de créations d'entreprises en France. Son but était de comparer les évaluations portées à trois reprises sur les mêmes projets sur une période de deux ans, de manière à tester la qualité du processus d'évaluation.

Cette étude montre que peu d'éléments s'étant révélés importants après coup avaient été négligés dans l'évaluation au départ, mais qu'il y a eu souvent un fort dérapage dans le temps.

L'évaluation du plan d'affaire et l'existence d'une vision claire du projet par le créateur ont toutes deux fourni de bons éléments annonciateurs du succès. Par contre, le jugement sur les capacités personnelles de l'entrepreneur a été plusieurs fois démenti par les faits.

Des conséquences pratiques sur les méthodes d'évaluation de projets de créations d'entreprises peuvent être tirées de cette étude, bien que d'autres recherches soient encore nécessaires. 


\title{
Notes
}

\section{De l'évaluation des projets de création d'entreprises au pronostic de succès}

\author{
Yves-Frédéric LIVIAN* \\ Groupe ESC Lyon \\ Stéphane MARION** \\ Groupe ESC Lyon
}

\begin{abstract}
RÉSUMÉ
On sait la difficulté de dégager des critères d'évaluation de projets de créations d'entreprises ayant une valeur de pronostic.

Cette note présente les résultats d'une étude portant sur un échantillon de 11 cas de créations d'entreprises en France. Son but était de comparer les évaluations portées à trois reprises sur les mêmes projets sur une période de deux ans, de manière à tester la qualité du processus d'évaluation.

Cette étude montre que peu d'éléments s'étant révélés importants après coup avaient été négligés dans l'évaluation au départ, mais qu'il y a eu souvent un fort dérapage dans le temps.

L'évaluation du plan d'affaire et l'existence d'une vision claire du projet par le créateur ont toutes deux fourni de bons éléments annonciateurs du succès. Par contre, le jugement sur les capacités personnelles de l'entrepreneur a été plusieurs fois démenti par les faits.
\end{abstract}

Des conséquences pratiques sur les méthodes d'évaluation de projets de créations d'entreprises peuvent être tirées de cette étude, bien que d'autres recherches soient encore nécessaires.

* M. Livian est docteur en sociologie. Professeur au Groupe École supérieure de commerce de Lyon, il est directeur de recherches à l'Institut de recherche de l'entreprise où il travaille principalement dans le domaine de la gestion des ressources humaines.

** M. Marion est titulaire d'un diplôme d'Études approfondies en sciences économ:ques. Il mène des recherches dans le domaine de la création d'entreprises au Centre des entrepreneurs du Groupe ESC Lyon. 


\begin{abstract}
It is well known that designing criteria for new business ventures evaluation having a predictive value is a very difficult task.

This paper presents the results of a study on a sample of eleven cases of new business ventures in France. Its purpose was to compare evaluations made on the same ventures at three moments in time over a two year period, in order to test the quality of new business ventures evaluation.

This study shows that few elements which appeared as critical afterwards had been neglected in the first evaluation, but that a "time skidding " phenomenon occurred frequently.

The evaluation of the Business Plan and the existence of a clear vision of the business by the entrepreneur, have both given good elements for success prediction. On the other hand, the judgment made on the entrepreneur's personal capacities has been denied by the facts on several occasions.

Practical consequencies on evaluation methods can be drawn from this study, although further research is necessary.
\end{abstract}

\title{
RESUMEN
}

Sabemos que es muy dificil encontrar criterios de evaluación de proyectos de creación de empresas que podrian anticipar el éxito de las mismas.

La nota presenta los resultados de un estudio llevando sobre un muestrario de 11 casos de creación de empresas en Francia. Su objetivo era de comparar las evaluaciones realizadas tres veces sobre los mismos proyectos en un período de dos años, a fin de probar la calidad del proceso de evaluacion.

Este estudio demuestra que pocos elementos que se han revelado importantes despues, habian sido ignorados en la evaluación al principio. Pero muchas veces hubo retrasos incontrolados.

La evaluación del plan d'affaire, y la existencia de una visión clara del proyecto por el creador han proporcionado buenos elementos que anticipan el éxito. En cambio, el juicio sobre las capacidades personales del empresario unas veces fue desmentido por los hechos.

Se puede sacar del estudio conclusiones prácticas sobre los métodos de evaluación de proyectos de creación de empresas, aunque otras investigaciones sean necesarias. 


\section{Introduction}

Au-delà de l'inévitable question sur les facteurs clés de succès de la création d'entreprises, s'est développée, plus récemment, une interrogation sur la capacité réelle des processus de sélection de projets à anticiper ces facteurs sur des projets précis. Comme dans d'autres domaines, ce passage de l'analyse «expost» - celle qui indique, après coup, ce qui a «fait la différence »- à la préconisation de critères ayant une valeur de pronostic est très difficile.

Parmi d'autres, des travaux récents, comme ceux conduits par P. Dubini (1989 et 1990), insistent d'ailleurs sur ces difficultés. Ils soulignent, d'une part, la spécificité des critères relatifs à la création d'entreprises - du fait de la complexité, de la dynamique et de la rapidité des changements à l'intérieur du processus - et, d'autre part, la prédominance des recherches se référant au processus de sélection des sociétés de capital de risque.

À partir de ces dernières, Ooghe (Ooghe et al., 1988) propose une répartition en deux groupes des critères utilisés dans l'analyse de projets. Le premier est composé par l'ensemble des critères relatifs à l'analyse de l'entrepreneur ou de l'équipe et des compétences en management réunies autour du projet. Le second regroupe les critères liés à l'analyse de l'activité décrite normalement dans un plan d'affaire.

L'importance donnée aux facteurs du premier groupe a été mise en évidence par Mac Millan, Siegel et Narashima (1985) dans une étude des facteurs utilisés par les sociétés de capital-risque, pour évaluer un projet. Parmi les dix critères relevés comme les plus significatifs, cinq étaient en relation directe avec l'expérience et la personnalité de l'entrepreneur ou de l'équipe.

Stuart et Abetti (1988) distinguent trois grands domaines dans la composition de ce premier groupe :

- l'analyse de la personnalité ;

- l'étude des connaissances accumulées (l'expérience);

- l'examen des caractéristiques du management, correspondant aux capacités et à la vision du créateur ou de l'équipe.

Sur le premier point, les recherches conduites pour distinguer un profil type de créateur à fort potentiel de réussite ont souvent eu des résultats limités. Ainsi, des études basées sur l'utilisation de différentes batteries de tests psychologiques (Sexton et Bowman, 1986; Livian, 1989 ; Beverina, 1989) ou d'enquêtes établies à partir d'échantillons significatifs (Begley et Boyd, 1987), ne distinguent que très partiellement les créateurs d'autres populations. Les conclusions s'arrêtent souvent à la constatation d'un plus fort besoin de se 
réaliser, d'une capacité plus importante à prendre des risques, d'une plus grande ténacité et d'une plus grande propension à accepter l'incertitude chez les créateurs que chez les non-créateurs.

Sur le second point souligné par Stuart et Abetti, de nombreuses études ont cherché à mettre en évidence des corrélations entre la scolarité, l'âge, le sexe, l'expérience, les différents modèles de référence du créateur et le succès de la création. Cooper, Dunkelberg et Woo (1988) ont fait dans ce sens un travail très intéressant à travers une étude longitudinale, sur 3 ans, de 2994 entreprises. Leurs résultats montrent, par exemple : que les hommes réussissent mieux que les femmes ou les créateurs issus de minorités; que les créateurs avec une expérience professionnelle et des études supérieures ont de meilleurs résultats ; qu'une expérience initiale en management n'est pas corrélée avec une forte probabilité de réussite alors qu'une expérience technique l'est. Ce rôle de l'expérience initiale se retrouve dans beaucoup d'autres études (Neiswander et Drollinger, 1986; Stuart et Abetti, 1987) et semble véritablement paraître comme un élément clé.

Sur le troisième point, dans la lignée des travaux initiés par Smith, tout un ensemble de recherches à caractère typologique (Peterson et Smith, 1986 ; Smith et al. 1987; Woo, Cooper et Dunkelberg, 1988) essaient d'associer types d'entrepreneurs et types d'entreprises. Sur ce troisième point, d'autres travaux montrent les relations qui peuvent exister entre la croissance de l'entreprise et les différents réseaux auxquels l'entrepreneur peut avoir accès (Birley, 1985; Aldrick, Rosen et Woodward, 1987 ; Ward et Randall, 1989). Sur un autre plan, plusieurs recherches mettent l'accent sur l'importance de donner à la démarche stratégique et à la structure du secteur à l'intérieur duquel l'entreprise est amenée à se développer (Sandberg et Hofer, 1987 ; Steiner et Solem, 1988).

Ce dernier point nous permet d'entrer directement dans le deuxième groupe de facteurs d'analyse défini par Ooghe, à savoir, ceux qui concernent l'étude de l'activité. Celle-ci suppose pratiquement l'existence d'un plan d'affaire. Ce document tend à se généraliser; il a d'ailleurs retenu l'attention de nombreux chercheurs pour savoir quels étaient son intérêt et ses limites, en fonction de sa structure et de son contenu (Frank, Plashka et Roessl, 1988). À l'intérieur de celui-ci sont généralement abordées les caractéristiques du produit ou du service ; du marché ; du plan de financement et des performances financières; du management et des ressources humaines; de la stratégie et de l'organisation.

Stuart et Abetti (1987) définissent par exemple dans cette perspective, le succès initial d'une nouvelle entreprise comme fonction du marché, du degré d'innovation, de la stratégie, de l'organisation et des capacités de direction de l'équipe dirigeante. Ils notent ainsi qu'il est préférable d'avoir une stratégie et 
une structure reflétant les comportements des autres entreprises du secteur ou encore, d'être plutôt sur un marché stabilisé que sur un marché nouveau en forte croissance.

MacMillan, Zemann et Narashima (1987) distinguent trois grandes classes d'échecs. Outre les équipes d'entrepreneurs peu qualifiées et ayant des produits peu différenciés, on trouve : des équipes qualifiées mais se heurtant rapidement à la concurrence et n'ayant pas assez de résistance pour y faire face, et celles qui, ayant ces deux caractéristiques, finissent par perdre leur avantage initial en raison de l'absence de protection possible de leur projet.

La nature du couple produit-marché, le type de processus de fabrication, le degré d'innovation et le degré d'inexpérience du créateur sont aussi avancés comme des facteurs déterminant le niveau de risque encouru (Massacrier et Rigaud, 1984 ; Gorman et Shalman 1984 ; Rigaud 1987 ; Ramus 1989).

Les indicateurs de performances économiques et financières sont également fréquemment évoqués. Différents auteurs soulignent par exemple l'importance de l'évaluation du risque relatif au manque de liquidité du fait, d'une part, de la tendance à la sous-capitalisation et, d'autre part, des écarts généralement rencontrés entre les prévisions et les réalisations (Fourcade, 1984 ; Rigaud, 1987 ; Neiswander et Drollinger, 1986).

Malgré tout l'intérêt et l'apport de ces recherches, il reste difficile de faire le lien entre ces risques ou ces critères de succès et les pratiques concrètes d'évaluation (Colombo, 1989 ; Depperu, 1989 ; Ramus, 1989).

Nous avons voulu travailler dans ce sens en essayant de voir en quoi les pratiques de sélection et de suivi de projets utilisées à l'entrée et au cours d'un programme d'appui à la création d'entreprises ${ }^{1}$ avaient pu avoir une vertu de pronostic. Pour ce faire, nous avons comparé les résultats de ces évaluations avec le devenir réel d'un groupe de projets analysés 12 à 18 mois plus tard.

1. Il s'agit du Programme d'appui (PA) du Groupe ESC Lyon créé en 1985 et qui accueille une trentaine de projets par an. 


\section{Présentation des objectifs de l'étude et des projets étudiés}

\subsection{Objectlfs de l'étude}

Le but de l'étude était de déterminer quel degré de prédiction avait été obtenu dans le processus de sélection et d'accompagnement des projets, en comparant les appréciations faites sur des projets au cours du programme d'aide et la réalité de ce qu'il en est advenu 12 à 18 mois plus tard.

L'analyse qui peut être faite sur ces projets ne peut conclure, après un an à un an et demi de lancement, à un succès définitif. Notre analyse cherche seulement à comprendre ce qui s'est passé réellement pendant les 18 premiers mois de leur activité par rapport aux évaluations faites au début par des spécialistes, au vu d'un dossier complet et d'entretiens avec l'entrepreneur.

Plus précisément, cette étude reposait sur un certain nombre d'hypothèses, tirées à la fois des publications écrites et de l'expérience pratique des évaluateurs, sur les points clés à analyser dans un projet.

Ce que nous voulions vérifier était essentiellement :

- la qualité globale du pronostic de viabilité des projets, durant leur phase de démarrage, en s'interrogeant sur la situation de ces projets un an à un an et demi après leur sortie du programme, c'est-à-dire de l'ordre de 18 à 24 mois après leur première formulation, et notamment la concrétisation du projet, l'évolution de son contenu, et le respect du calendrier fixé ;

- le rapport qui pouvait exister entre l'évaluation du plan d'affaire fait à la fin du programme et la situation de l'entreprise creee 12 à 18 mois après ;

- l'existence de facteurs ou d'événements imprévisibles et leur influence sur le démarrage du projet ;

- la qualité de l'évaluation qui avait été faite des caractéristiques personnelles du créateur d'entreprise, en comparant l'analyse faite au départ avec les éléments personnels constatables au cours de la période de lancement ;

- le rôle joué dans le déroulement de la phase de démarrage par le caractère plus ou moins innovateur du projet. 


\subsection{Méthodologie}

Le choix s'est porté sur une méthodologie permettant de suivre l'évolution du projet et utilisant les données recueillies tous les ans auprès des créateurs ayant suivi ce programme d'aide.

Ce genre d'étude longitudinale, assez rare dans les publications écrites, a l'avantage de permettre un jugement sur des évolutions et non pas se borner à une vision instantanée, même si elle ne peut que porter sur des échantillons réduits.

Onze projets ont été choisis par tirage au sort en mai 1987, sur une population totale de $36^{2}$. Les créateurs auteurs de ces projets ont participé au Programme d'appui $n^{\circ} 3$ qui s'est déroulé en 1986 ( 3 projets), aux Programmes d'appui nos 4 et 5 qui se sont déroulés en 1987 (4 projets chacun).

Huit projets concernent une activité de services, trois une activité industrielle. Pour chacun d'entre eux, nous avons effectué l'analyse de trois évaluations :

- L'évaluation faite à l'entrée du Programme par l'équipe d'évaluateurs, sur la base des dossiers remplis lors de la session d'admission et d'un entretien avec l'entrepreneur. Ce dossier comporte cinq rubriques d'analyse sur l'entrepreneur et son entourage, quatre sur le projet luimême et une synthèse sur "opportunités et menaces particulieres $d u$ projet" (voir annexe 2).

- L'évaluation portée par les responsables du Programme sur le plan d'affaire remise par chaque createur à la fin du Programme (environ six mois après leur entrée) et présentée au cours d'une séance à un jury composé des responsables du Programme et de banquiers susceptibles d'être intéressés. Ces appréciations étaient consignées dans des dossiers, qui ont été systématiquement étudiés ${ }^{3}$.

- Les données recueillies sur chaque entreprise un an à un an et demi après cette date, grâce à la banque de données mise en place au Centre des entrepreneurs du groupe ESC Lyon et à un entretien réalisé avec le créateur.

2. Sur une population-mère de 36 projets, on a éliminé du tirage ceux dont le succès paraissait trop lié à un seul facteur-« véto » et ceux qui correspondaient à une entreprise déjà constituée. Restaient 25 projets, sur lesquels un tirage au sort a eté effectué.

3. L'annexe 3 présente la grille d'analyse utilisée pour les plans d'affaire. 
Nous décrivons sommairement ci-dessous quelques caractéristiques des 11 projets et leur situation après 18 mois.

- BC est une entreprise d'édition et d'impression sur textile reposant sur un concept original. Elle est lancée par une personne seule, mais disposant d'un réseau de relations important dans le domaine de ses produits.

Une ambitieuse campagne de promotion s'avère un lourd échec financier. L'entreprise dépose son bilan après 18 mois d'activité.

- PM est une entreprise de conception et réalisation d'éléments en bois, destinés aux collectivités, lancée par une personne ayant déposé un brevet sur le procédé de fabrication.

L'entreprise, après un démarrage relativement lent, poursuit son activité.

- JC est un projet de distribution originale dans le domaine agro-alimentaire qui n'a pas pris corps.

- GV est une entreprise de recherche et développement sur des produits de haute technologie, fondée par deux partenaires ayant une expérience professionnelle dans le domaine.

Démarrant plus tard que prévu, l'entreprise poursuit son activité et se développe.

- FD est une entreprise de services aux entreprises lancée par une personne sur une idée innovante. Son démarrage est plus lent que prévu et elle poursuit son activité s'étant légèrement diversifiée.

- AL est une entreprise de conseil en informatique industrielle lancée par une personne seule et poursuivant son activité.

- ES est une entreprise de distribution du produit d'un fournisseur, lancée par une personne seule et ayant déposé son bilan après six mois d'activité.

- SV est une entreprise de fabrication et de vente d'un produit innovant pour l'habitat lancée par deux personnes et qui se développe.

- SI est une entreprise de services, élaborant et vendant des logiciels spécialisés sur un marché nouveau, lancée par une personne seule et poursuivant son activité.

- DB est un projet d'entreprise de service informatique qui n'a pas été lancé. 
- MM est une entreprise de conseil et de services aux entreprises lancée par une personne seule et qui poursuit une activité largement différente du projet initial.

L'annexe 1 résume certaines caractéristiques des projets et fournit les chiffres disponibles.

\section{Constats globaux sur les projets}

\subsection{Bllan de créations contrasté mais peu surprenant}

Tout d'abord un premier constat s'impose : sur les 11 projets tirés au sort, 2 n'ont pas abouti.

- Le projet DB n'a pas débouché sur une création d'entreprises. L'évaluation était positive sur la personne du créateur, considéré comme sérieux et compétent mais celui-ci n'a pas réussi à pénétrer le marché, s'est découragé devant les difficultés et a abandonné.

- Le projet JC, fondé sur une activité mono-fournisseur, s'est heurté à une élévation du prix des produits à distribuer. L'évaluation avait repéré la vulnérabilité du projet, mais le produit paraissait bon et le créateur capable.

Sur les neuf entreprises créées, deux ont déposé leur bilan : ES et BC. Restent donc en activité sept entreprises, dont une réalise aujourd'hui une activité différente du projet initial. Le taux d'échec n'est pas surprenant et est même inférieur à ceux généralement avancés.

Ce qui frappe dans la comparaison entre le devenir des projets et les évaluations initiales, c'est que les éléments d'évaluation pris en compte dans le processus d'évaluation du Programme d'appui couvrent assez largement la réalité observable avec le recul du temps.

Plusieurs remarques peuvent en effet être faites dans ce sens.

Tout d'abord, dans les deux cas de non-aboutissement, l'acceptation du produit par le marché - à certaines conditions de prix dans le second cas - a été l'élément déterminant et avait été perçue dans l'évaluation bien qu'à des degrés divers. Le risque encouru par JC était visible : on ne pouvait simplement pas prévoir les évolutions de prix ; mais à partir du moment où le risque de dépendance existait, la question essentielle posée par le projet était l'absence d'alternative possible. Pour DB, il est vrai, cette difficulté avait été moins fortement évaluée, et ce qui apparaît peut-être comme une insuffisante ténacité du créateur, n'avait pas été pressenti. 
Ensuite, un certain nombre d'obstacles rencontrés réellement par les créateurs avaient été repérés par les évaluateurs. Par exemple, les difficultés actuelles rencontrées par AL dans le domaine commercial, liées à un manque d'intérêt pour la vente avoué par le créateur lui-même lors de l'entretien, correspondent bien à un jugement porté dès le début par les évaluateurs. La dépendance de JC vis-à-vis d'un partenaire a également été perçue et des réserves importantes avaient été faites sur la clarté et l'ambition du projet. D'autre part, plusieurs des raisons essentielles de deux « échecs » correspondent à des éléments repérés, voire diagnostiqués dès l'entrée dans le Programme comme en témoignent les dossiers qui ont été analysés. Ainsi, l'absence d'ambition et de prise de risque observée par les évaluateurs dans le projet ES était dès le début considérée comme un point d'interrogation sérieux, ainsi que l'écart entre le potentiel de l'individu (directeur marketing d'un important groupe agroalimentaire) et la taille du projet.

L'importance, sans doute exagérée, de l'enjeu que constituait la première opération de vente à l'export de $\mathrm{BC}$ a aussi été soulignée avant sa réalisation, ainsi que la relative fragilité de la construction financière du projet.

De même, l'analyse "un an après " révèle peu d'éléments réellement inopinés. Bien entendu, certains phénomènes se sont accélérés, ou au contraire les créateurs estiment avoir sous-évalué certains délais. On ne trouve cependant pas, pour l'instant, de facteurs importants ayant été décisifs pour l'évolution du projet et qui auraient été d'une autre nature que ceux évoqués dans l'évaluation.

Cela veut-il dire pour autant qu'on aurait enfin découvert une méthode d'évaluation infaillible? Bien sûr que non. D'abord parce qu'il y a eu une relative surprise, comme celle d'un succès après une évaluation réservée (projet GV). Ensuite, ce qui parait davantage en jeu est plutôt le rythme et l'importance relative des différents facteurs d'évolution du projet, concourant dans tous les cas à un développement de l'entreprise décalé dans le temps par rapport aux prévisions ${ }^{4}$.

On retrouve dans la nature du jugement sur les projets des analogies avec les problèmes du diagnostic et du pronostic médical: une certaine structure de facteurs est repérable, et permet de formuler dans un grand nombre de cas, grâce à un savoir-faire empirique, un diagnostic valable.

La tâche consistant à faire un pronostic est d'une autre nature, car elle doit tenir compte : de la spécificité des cas, d'un pari sur le temps, de la résistance du sujet ... On a repéré des symptômes, des risques potentiels : si la

4. D'ailleurs, comme on le voit dans l'Annexe 1, sur les 7 projets encore vivants, 5 semblent, d'ores et déjà, parvenir à des volumes d'affaires en-deça des prévisions, quelquefois dans des proportions considérables. 
personne tombe malade, on saurait presque dire de quelle maladie il s'agira ... Mais voilà : tombera-t-elle malade ? Et quand ? Là s'intercalent des facteurs contingents qui rendent difficile un pronostic sûr, même sur la base d'un diagnostic révélant l'essentiel ${ }^{5}$.

\subsection{Dérapages dans le temps}

Un autre point remarquable est que le démarrage est presque toujours plus difficile que prévu. Bien qu'il y ait peu d'éléments réellement imprévus dans leur nature, les cas sont nombreux où certaines difficultés pressenties ont atteint une plus grande ampleur qu'il n'était imaginé. La négociation avec les banques (BC, FD), la durée de la décision d'achat (AL, PM), la difficulté de recruter et de constituer une équipe (FD, PM) en constituent des exemples marquants.

Là encore, c'est moins la nature de l'obstacle que sa hauteur ou le temps nécessaire pour le franchir qui surprennent.

FD considère qu'il a fallu d'abord créer le marché de son service, et admet avoir sous-estimé la durée de son lancement. Le cas de SI est très proche. PM a sous-estimé la lenteur du processus de décision d'achat. GV a sous-estimé le temps nécessaire au recrutement de collaborateurs adaptés.

Cette remarque renforce l'importance de la ténacité et de la motivation comme critères d'évaluation personnelle du créateur, comme l'ont signalé de nombreux chercheurs et praticiens.

"C'était prévu (que cela serait difficile), mais ça l'est, en fait, encore plus ! » déclarent presque dans les mêmes termes deux créateurs interrogés.

L'importance des dérapages dans le temps est d'ailleurs confirmée par l'étude de J. Rigaud (1987) sur les conditions du démarrage.

L'adéquation des ressources financières pour faire face aux frais fixes incompressibles devient donc un élément de pronostic d'autant plus important que ces frais sont élevés et que les risques de dérapage sont importants (produit innovant, marché à créer, décisions d'achat longues et soumises à des facteurs saisonniers ou " politiques », etc.).

5. Cette observation est à rapprocher de celles émises par certains chercheurs soulignant que la création d'entreprises est toujours une expérience, fondée sur des hypothèses relatives aux relations entre produits, marchés et concurrents, certains allant jusqu'à dire qu'il n'est possible de les tester ... qu'en lançant l'entreprise. Les chercheurs soulignent aussi l'importance de l'information recueillie à chaque étape de la création (et qu'il n'est pas possible d'avoir avant). $C f$. Block et MacMillan (1985). 


\subsection{Importance de l'évaluation du plan d'affaire}

L'étude effectuée sur notre échantillon montre que l'évaluation du plan d'affaire fournit un élément très utile de jugement sur le projet, même si, bien sûr, il n'est pas suffisant.

Telle qu'elle a été définie par l'équipe d'évaluateurs, l'évaluation du plan d'affaire met en évidence la cohérence du projet, son orientation stratégique, sa force de conviction à l'égard d'éventuels bailleurs de fonds. Ses qualités formelles peuvent aussi être considérées comme un indice de qualité de raisonnement et de communication.

Or, il est à noter que sur les quatre "non créateurs ", trois avaient fait l'objet d'appréciations mauvaises de leur plan, et un avait fait l'objet de réserve sur la cohérence de son plan.

Le plan d'affaire de GC est considéré comme "flou ", sans références objectives, le dossier financier est jugé très faible. Celui de JC fait l'objet d'une appréciation très réservée, "manquant d'ambition » et peu clair. Celui de DB est jugé " seulement descriptif », "creux et sans âme ». Seul, celui de ES fait l'objet d'une évaluation acceptable, avec des réserves.

De la même manière, il y a convergence, en positif, pour SI, SV et PM dont les plans étaient jugés bons. Bien entendu, cette analyse n'est pas totalement prédictive, puisqu'un des projets à s'être développé avait fait l'objet, à l'origine, d'une mauvaise évaluation de son plan (GV) et que certains projets dotés d'un excellent plan connaissent actuellement des difficultés.

Cependant, on peut retenir que les critères d'appréciation utilisés permettent de mettre en évidence des qualités du projet qui constituent une base indispensable. Ceci ne veut pas dire que le créateur d'entreprise qui réussit est celui qui fait des plans détaillés et soignés. Mais le fait que l'existence de plans détaillés ne caractérise pas les entrepreneurs qui réussissent, en phase de croissance, n'invalide pas pour autant l'examen du plan d'affaire comme un élément de jugement, surtout au moment même du lancement. On peut utiliser le plan d'affaire au début d'une création, comme un exercice révélant ou non certaines qualités du projet. C'est presque moins son contenu qui apporte un élément annonciateur que les capacités manifestées par le créateur, surtout si celui-ci sait faire évoluer le plan dans le temps, au fur et à mesure du mûrissement du projet.

On rejoint ainsi les conclusions de certaines études antérieures, qui insistent davantage sur la qualité du plan d'affaire que sur son étendue et sa structure. Il importe de juger des capacités du créateur à distinguer l'important de l'accessoire. L'exercice du plan d'affaire peut être un outil contribuant à l'évaluation de la capacité stratégique du créateur. 
On parvient ainsi à une autre remarque relative à la vision possédée par le créateur. Dans les deux cas de non-création, dans un cas d'échec sur deux, et au moins un cas de réussite à fort potentiel, il apparaît que l'existence, par le créateur, d'une «vision " claire de l'avenir de son projet peut être considérée comme un critère pronostique possible.

Sans sombrer dans une conception "visionnaire " de l'entrepreneur, à la mode aujourd'hui aux États-Unis, cette notion correspond suffisamment à d'autres recherches (notamment celles qui définissent le leadership à travers cette caractéristique) pour mériter d'être retenue ${ }^{6}$.

La capacité de se représenter l'avenir de son projet, avec suffisamment de précision et de clarté, tant dans sa dimension stratégique que dans certains éléments quotidiens, peut être en effet un atout considérable, puisque cela peut permettre d'anticiper, de choisir et de convaincre. JC, DB, deux cas d'échecs, avaient été jugés défavorablement par rapport à cette capacité.

Bien entendu, l'existence ou non de cette vision est plus importante à certaines phases du projet qu'à d'autres (pour passer du projet ouvert au projet finalisé, par exemple). Mais il apparaît néanmoins que toute expression du créateur qui l'amène à formuler une représentation de son entreprise peut éclairer notablement les évaluateurs.

\subsection{Difficultés de l'évaluation du créateur}

L'évaluation du créateur lui-même a joué un rôle central dans l'appréciation portée sur les projets. On le constate tout d'abord par le fait que, dans certains cas, c'est avant tout la personne qui a suscité l'intérêt du jury.

L'analyse des dossiers d'évaluation des projets révèle, en effet l'importance du poids du créateur lui-même dans l'examen d'ensemble du projet. Dans 5 des 11 cas, l'intérêt porté au créateur a été supérieur à l'intérêt porté au projet, tandis que le projet a semblé l'emporter sur la personne dans seulement deux cas.

Cet intérêt n'est pas anormal étant donné les préoccupations pédagogiques (Programme d'appui à la création d'entreprises) et le fait que beaucoup d'admissions dans ce programme se font à un stade très «amont » du projet. Toutefois, il faut bien reconnaître que cette évaluation expose à bien des aléas : dans au moins trois cas de non-création ou d'échecs sur quatre, le jugement sur la personne s'est avéré largement positif.

6. Par exemple, l'étude de Van den Ven, Huston et Schroeder (1984), qui indique qu'une "idée claire et large de son affaire » est l'un des éléments qui différencient les entrepreneurs performants des autres. 
Pour DB, le créateur était jugé à l'origine sérieux et compétent. Le créateur de ES était vu comme doté d'une grande expérience ; on doutait seulement de sa capacité à travailler en équipe. Le créateur de BC, quant à lui, a paru " déterminé », compétent dans son domaine et très " motivé ".

On notera que dans ces cas, certains mécanismes inhérents à l'évaluation en face à face ont joué et peuvent expliquer ce qui apparait après coup comme des « erreurs » de jugement :

- une certaine séduction de la personne sur les évaluateurs (cas du projet $\mathrm{BC})$. On retrouve également une situation proche dans les évaluations de FD et SI ;

- une certaine apparence de compétence et de sérieux qui a pu être « travaillée » par le candidat se préparant aux entretiens (cas de DB, et peut-être de ES).

Ce constat soulève le problème du rapport entre l'évaluateur et le créateur "évalué », et celui de la qualité des relations personnelles entre eux. L'intérêt de l'évaluateur pour le projet et le « halo technologique » dont celui-ci est entouré aux yeux de l'évaluateur peuvent grandement perturber le jugement et le faire s'éloigner de l'impartialité requise ${ }^{7}$.

À l'inverse, deux créations intéressantes se sont faites avec un créateur faisant l'objet, au moins au départ, de réserves ou de désaccords entre évaluateurs sur ses caractéristiques personnelles (GV, SV). Ce constat souligne le caractère évolutif des phénomènes humains relatifs à la création et met en lumière un élément essentiel, qui est la capacité d'apprentissage du créateur.

Sur plusieurs cas, ce qui a été observé ou ce qui est encore douteux est moins l'ensemble des caractéristiques personnelles du créateur que sa capacité, à partir de ces caractéristiques (quelles qu'elles soient), à évoluer d'une manière positive (on revient à l'analogie médicale évoquée plus haut).

Les créateurs de GV et SV paraissent avoir évolué favorablement, alors que ES peut-être pas assez. Le créateur $\mathrm{AL}$ fait l'objet d'un jugement réservé et persistant sur ses capacités commerciales. Quant au créateur de BC, pouvait-il acquérir certaines capacités complémentaires qui lui auraient permis d'éviter l'échec ? Le créateur de MM, quant à lui, reconnaît n'avoir pas tenu compte de certains conseils et explique lui-même certaines difficultés qu'il a éprouvées par son « refus d'évoluer».

Dans tous ces cas, l'élément décisif du pronostic est la capacité d'apprentissage et d'évolution du créateur.

7. Ces points sont développés dans le texte de V. Ramus (1989). 
Ce pronostic est donc largement facilité lorsque l'on voit évoluer le créateur entre deux entretiens ou pendant le programme pédagogique considéré, étant entendu que ce délai d'observation permet aussi de tester l'efficacité du créateur dans la résolution des problèmes qu'il rencontre.

Un autre aspect individuel confirme son importance et est, lui, plus facile à juger: il s'agit de l'expérience professionnelle antérieure du créateur. Les résultats sur notre échantillon vont bien dans le sens des recherches antérieures : l'un des cas d'échecs est celui d'une personne qui, bien que qualifiée et disposant d'appuis, n'avait pas une expérience précisément centrée sur l'activité. À l'inverse, la seule entreprise dont le chiffre d'affaires actuel est au-delà des prévisions initiales, GV, a été fondée par deux partenaires spécialisés depuis longtemps dans le domaine. Une autre entreprise en bonne santé, est dans ce cas.

\subsection{Difficultés particulières de l'innovation}

Une autre hypothèse de recherche, confirmée par nos observations, réside dans la difficulté particulière des produits les plus innovants. Bien qu'il soit toujours difficile d'établir une hiérarchie dans l'innovation, il apparaît que certains projets de notre échantillon, ayant en commun un plus haut degré d'innovation, ont connu des difficultés plus importantes que ce qui avait été prévu quant à leur accès au marché.

SI, et dans une certaine mesure SV, ont expérimenté à leurs dépens l'importance de la notoriété et la difficulté à entrer sur un marché fortement structuré. Dans ces cas, les techniques de ventes classiques se révèlent inadéquates pour pénétrer un marché dont le potentiel n'est cependant pas remis en cause. FD et AL éprouvent également des problèmes du même ordre.

Cette observation souligne l'importance, pour les projets les plus innovants, du réseau de notoriété établi auparavant par le créateur et de la solidité des appuis financiers dont il doit disposer pour faire face à une période longue de pénétration du marché.

En dehors de ces difficultés d'accès au marché, d'autres problèmes sont apparus plus en amont dans la mise au point des produits et dans le passage à la fabrication de séries.

$\mathrm{SV}$, qui avait fabriqué ses prototypes dans des conditions artisanales a ainsi connu des difficultés dans la réalisation de séries. La mise en place d'un processus industriel entraîne un certain nombre de contraintes qui n'ont pas été perçues lors de l'élaboration du prototype. De même, le recours partiel à une sous-traitance qui n'apporte pas toutes les satisfactions attendues, en terme de qualité et de délai, est un facteur pénalisant initialement sous-estimé. 
SI et GV, pour lesquels un travail de recherche et développement important reste à faire, connaissent des écarts importants dans la mise au point des produits commercialisables. Cependant la mise en place d'une activité de service, à partir des premiers développements, apparaît dans ces cas comme un palliatif permettant de supporter ces dérapages. Ces deux constats sur la mise au point des produits et sur le passage à un processus de fabrication industriel, confirment toute la difficulté qui existe à mesurer l'état d'avancement d'un produit et surtout à évaluer le délai nécessaire entre ses derniers perfectionnements et sa mise sur le marché. Nous avons ici une des principales causes d'écarts entre les prévisions et les réalisations évoquées précédemment.

\section{Conclusion}

Bien sûr, on ne peut tirer d'une étude portant sur un petit nombre d'entreprises des conclusions générales sur les conditions de succès des entreprises en création. Par contre, l'étude de la qualité prédictive d'une méthode d'évaluation (même si elle ne prétend pas à l'infaillibilité) permet, croyons nous, de dépasser l'analyse ex-post des critères de réussite et de dégager quelques remarques intéressantes quant aux pratiques d'évaluations de projets.

En utilisant un assez grand nombre de critères d'analyse relatifs au projet, à son environnement, à la relation entre le projet et l'individu ou l'équipe qui le porte, et en accumulant une expérience d'évaluateur, il est relativement facile de détecter les principaux points forts et points faibles du projet. Dans notre étude, la qualité prédictive de la méthode utilisée, si elle était analysée dans ce sens, serait très bonne. Les éléments commerciaux notamment, considérés comme déterminants, ont été dans l'ensemble analysés avec justesse. Par contre, l'entreprise en création est un système en équilibre fragile, ayant une dynamique pouvant donner une importance variable dans le temps à l'un ou l'autre des éléments (qualité commerciale, importance des investissements, capacité de réaction des hommes ...). C'est l'évolution de cet équilibre dynamique qui est difficile à prévoir.

Pour passer d'une "bonne " évaluation à un pronostic valide, il faut pouvoir prendre en compte l'évolution du projet dans le temps.

Ceci plaide donc pour des processus dynamiques d'évaluation, capables, non seulement d'analyser la valeur des différents critères, maintenant bien connus, à un moment donné, mais d'en observer la structuration à plusieurs reprises, en intégrant complètement la dimension temps. Dans cette perspective, on ne peut que déplorer la faiblesse des recherches sur l'évaluation des entreprises en création ayant pris en compte le rythme de maturation du projet. 
Cette prise en compte du temps pourrait être développée par des dispositifs d'évaluation diachroniques. Elle le serait aussi par une accentuation de l'étude de deux dimensions qui pourraient bien être à valeur prédictive: l'adéquation des ressources financières, pour faire face aux fréquents dérapages, et la capacité d'apprentissage ainsi que la ténacité du créateur.

L'évaluation des caractéristiques personnelles du créateur pose, quant à elle, de redoutables problèmes. Les capacités du créateur sont déterminantes mais doivent surtout être examinées à la lumière de son expérience professionnelle, en complétant le jugement ponctuel procuré par l'étude du dossier par une vision diachronique qui peut permettre de tirer des enseignements sur son potentiel personnel d'évolution. Ce constat confirme l'importance que l'on doit accorder à l'expérience du créateur ou de son équipe.

De même, les effets perturbateurs du jugement sur la personne que nous avons abordés peuvent être partiellement compensés par une confrontation d'opinions diverses entre évaluateurs et par une formalisation assez poussée du processus d'évaluation (grilles d'analyse, comptes rendus d'évaluation, ....).

L'évaluation du plan d'affaire, et notamment de la « vision » qu'il révèle, de sa solidité, de sa mise en valeur des ressources du créateur et de ses qualités formelles, fournit un bon moyen d'étudier plusieurs éléments déterminants. Bien sûr, nous ne retenons pas l'hypothèse d'une capacité du créateur à prévoir avec justesse ce qui va se passer. De nombreuses études ont même montré qu'il n'existait pas de corrélation directe entre l'existence du plan d'affaire et le succès de la création. Il s'agit plutôt de considérer le plan d'affaire comme une occasion formelle d'évaluer certaines aptitudes du créateur pouvant avoir une valeur prédictive, comme la capacité de se représenter son projet et d'en fonder la cohérence. De plus, il permet de montrer la capacité de conviction du créateur vis-à-vis des partenaires dont il aura besoin dans l'avenir.

Bien entendu, ces conclusions mériteraient d'être approfondies. Nous avons toutefois considéré qu'elles pouvaient inspirer d'ores et déjà certaines améliorations dans les pratiques d'évaluation, que nous avons d'ailleurs commencé à appliquer dans notre institution.

Compte tenu du chemin qui reste à parcourir dans le domaine de l'évaluation de projets, il nous semble qu'un effort accru de recherches longitudinales devrait précisément porter sur les méthodes et pratiques concrètes de pronostic, seules susceptibles d'aider les « évaluateurs » dans leur difficile tâche. 
ANNEXE 1

Caractéristiques des projets

\begin{tabular}{|c|c|c|c|c|c|c|}
\hline Projets & $\begin{array}{l}\text { Nombre de } \\
\text { créateurs }\end{array}$ & $\begin{array}{l}\text { Nombre d'années } \\
\text { exp. prof. } \\
\text { activité }\end{array}$ & Activité de l'entreprise & $\begin{array}{l}\text { CA prévu } \\
\text { au B.P. } \\
\text { (en KF) }\end{array}$ & $\begin{array}{c}\text { CA réalisé } \\
\text { un an après } \\
(\text { en } K F)\end{array}$ & Remarques \\
\hline BC & 1 & 0 & $\begin{array}{l}\text { Edition et impression d'œuvres } \\
\text { artistiques }\end{array}$ & 1360 & 700 & $\begin{array}{l}\text { Dépôt de bilan } \\
\text { après } 18 \text { mois }\end{array}$ \\
\hline PM & 1 & 0 & Création et commercialisation & 3800 & 1600 & \\
\hline JC & 1 & Oui & Distribution agro-alimentaire & - & - & Abandon \\
\hline GV & 2 & 5 et 10 ans & $\begin{array}{l}\text { Recherche et développement } \\
\text { produits haute technologie }\end{array}$ & 4626 & 6900 & \\
\hline FD & 1 & 10 ans & Services aux entreprises & 6080 & $\begin{array}{l}\text { Non encore } \\
\text { disponible } \\
\text { mais infér. }\end{array}$ & \\
\hline AL & 1 & 0 & Conseil informat. indust. & 711 & 300 & \\
\hline ES & 1 & 10 & Vente produits agro-alimentaires & 2464 & - & $\begin{array}{l}\text { Dépôt bilan } \\
\text { après } 6 \text { mois }\end{array}$ \\
\hline SV & 2 & $\begin{array}{l}10 \text { ans pour } \\
\text { l'un des deux }\end{array}$ & $\begin{array}{l}\text { Fabrication et vente d'équipements } \\
\text { pour la maison }\end{array}$ & 4213 & Non disponible & \\
\hline SI & 1 & 5 ans & Conception et vente de logiciels & 1072 & 600 & \\
\hline DB & 1 & Oui & Service informatique & - & - & Abandon \\
\hline $\mathbf{M M}$ & 1 & 10 ans & Conseils services PME & 2464 & 700 & $\begin{array}{l}\text { Changement } \\
\text { d'activité }\end{array}$ \\
\hline
\end{tabular}


ANNEXE 2

\section{Canevas simplifié de la fiche d'évaluation utilisée à l'entrée du Programme d'aide}

1. LE CANDIDAT ${ }^{*}$

1. Capacités personnelles ( 7 facteurs);

2. Motivation (3 facteurs);

3. Attitude de l'environnement personnel (4 facteurs);

4. Qualité de l'équipe (5 facteurs);

5. Savoir-faire (7 facteurs).

\section{LE PROJET}

1. Attractivité, qualité du marché (4 facteurs);

2. Degré de connaissance du marché (6 facteurs);

3. Avantages concurrentiels du produit ( 3 facteurs);

4. Atouts du projet (6 facteurs).

\section{OPPORTUNITÉS ET MENACES PARTICULIERES DU PROJET}

\section{SYNTHÈSE}

1. Synthèse de l'évaluation portée sur le projet ( 3 critères);

2. Synthèse de l'évaluation portée sur le créateur ( 3 critères);

3. État d'avancement du projet ;

4. Principaux atouts et handicaps.

* Pour la majorité des facteurs proposés, on demande à l'évaluateur une note entre 1 et 4 . Un commentaire détaillé explique, pour certains facteurs, le sens qu'on doit y donner. 
ANNEXE 3

Recherche évaluation des projets de création d'entreprise

NOM :

ENTREPRISE :

\section{Grille d'évaluation du plan d'affaire}

1. Clarté de la vision du devenir de l'entreprise

Cohérence, précision, caractère évolutif

du projet

2. Solidité du plan d'affaire

Clarté de la stratégie explicite ;

Cohérence interne du B.P.

(Objectifs

marchés, produits, ressources

financières) ;

Information objective existante

(Produits, marchés).

3. Explicitation et valorisation des ressources humaines existantes

Lucidité dans l'exposé des ressources ;

Caractère convaincant de leur mise en valeur.

4. Explicitation des demandes de ressources

Capacité à convaincre de la nécessité

de l'appuyer

5. Qualité de la présentation

Rigueur ;

Précision ;

Qualité de la synthèse.

Appréciation générale : 


\section{Bibliographie}

Aldrich, H., B. Rosen et W. Woodward (1987), « The impact of social networks on business foundings and profits : a longitudinal study », dans Frontiers of Entrepreneurship Research, Babson College, p. 154-168.

Begley, T.M. et D.P. BoYD (1987), « Psychological characteristic associated with performance in entrepreneurial firms and smaller businesses ", Journal of Business Venturing, vol. 2, $\mathrm{n}^{\circ} 1$, p. 79-93.

BERRYMAN, J., "Small business failure and bankruptcy: a survey of the literature ", European Business Journal, vol. 1, $\mathrm{n}^{\circ}$ 4, p. 41-59.

BEVERINA, R. (1989), « The evaluation of entrepreneurial profiles », dans P. DuBIN (éd.), The Evaluation of Entrepreneurial Projects and Profiles, Milan, EGEA, p. 15-28.

BIRLEY, S. (1985), "The role of networks in the entrepreneurial process ", Journal of Business Venturing, vol. 1, $\mathrm{n}^{\circ} 1$, p. 107-118.

Block, Z. et I.C. MacMillaN (1985), " Milestones for successful venture planning », Harvard Business Review, 5.

Bowman-Upton, N., S.L. Scaman et D.L. Sexton (1989), «Innovation evaluation programs : do they help the inventors? ", Journal of Small Business Management, vol. $27, n^{\circ} 3$, p. $23-30$.

CAPIEZ, A. (1988), "Conditions et modalités d'émergence des TPE et PE ; l'exemple du Maine-et-Loire (France) », Revue internationale PME, vol. 1, $\mathrm{n}^{\circ}$ 2, p. 127-155.

Colombo, G. (1989), "The selection of entrepreneurial profile and projects », dans P. DuBin (éd.), The Evaluation of Entrepreneurial Projects and Profiles, Milan, EGEA, p. 1-14.

COOPER, A., W. Dunkelberg et C.Y. Woo (1988), « Survival and failure : a longitudinal study ", dans Frontiers of Entrepreneurship Research, Babson College, p. 225-237.

DEPPERU, D. (1989), «The selection of new entrepreneurial projects for training purposes », dans P. DuBin (éd.), The Evaluation of Entrepreneurial Projects and Profiles, Milan, EGEA, p. 73-92.

DuBiN, P. (1989), « The evaluation of new ventures and entrepreneurs : methodological considerations », dans P. Dubini (éd.), The Evaluation of Entrepreneurial Projects and Profiles, Milan, EGEA, p. 103-122.

Dubin, P. (1990), «Assessing new ventures success », dans S. Birley (éd), Building European Ventures, Amsterdam, Elservier science publishers B.V., p. 179-197.

Dubin, P. et I.C. MacMillan (1988), "The evaluation of entrepreneurial profiles in venture capital backed projects ", dans Frontiers of Entrepreneurship Research, Babson College, p. 46-58.

FouRCADE, C. (1984), «The « demarrage » of firms : international comparisons ", International Small Business Journal, vol. 3, $\mathrm{n}^{\circ} 2$, p. 20-32.

Frank, H., G. PlashKa et D. Roessl (1988), « Planning behavior of successful and nonsuccessful founders of new ventures », European Snall Business Seminar, GandBruxelles, $25 \mathrm{p}$.

Gorman, M. et W.A. Shalman (1986), «What do venture capitalists do?», dans Frontiers of Entrepreneurship Research, Babson College, p. 414-436.

GosLIN, J. et B. BARGE (1986), «Entrepreneurial qualities considered in venture capital support », Frontiers of Entrepreneurship Research, Babson College, p. 366-379.

HISRICH, R.D. et A.D. JANKOWICZ (1990), «Intuition in venture capital decisions : an exploratory study using a new technique », Journal of Business Venturing, vol. 5, $\mathrm{n}^{\circ} 1$, p. 49-62. 
KHAN, A.M. (1987), « Assessing venture capital investments with noncompensatory behavioral decision models ", Journal of Business Venturing, vol. 2, $\mathrm{n}^{\circ} 3$, p. 193-206.

Livian, Y.F. (1989), "An evaluation of the personal characteristics of new entrepreneurs, an empirical study ", dans P. Dubinı (éd.), The Evaluation of Entrepreneurial Projects and Profiles, Milan, EGEA, p. 29-42.

MacMirlan, I.C., R. Siegel et P.N. Subba Narashima (1985), « Criteria used by venture capitalist to evaluate new venture proposals ", Journal of Business Venturing, vol. $1, n^{\circ} 1$, p. $119-128$.

MacMillan, I.C., L. Zemann et P.N. SubBa Narashima (1987), « Criteria distinguishing successful from unsuccessful ventures in the venture screening process », Journal of Business Venturing, vol. 2, $n^{\circ} 2$, p. 123-138.

MARIon, S. (1990), «Évaluation de projets de création d'entreprises innovantes » Banque, 504, p. 406-412.

MASSACRIER, G. et G. RigAUd (1984), "Le démarrage d'activités nouvelles : aléas et processus », dans Revue française de gestion, mars-mai, p. 5-18.

NeIswander, D.K. et J.M. DrollINGER (1986), « Origins of successful start-up ventures », dans Frontiers of Entrepreneurship Research, Babson College, p. 328-334.

O'NeIL, H.M. et J. DUKER (1986), "Survival and failure in small business », Journal of Small Business Management, vol. 24, $\mathrm{n}^{\circ} 1$, p. 38-46.

Ooghe, H., C. van Wymersch, M. ERnst et P. van Den Bossche (1988), «Empirical analysis of the differences between successful and unsuccessful new enterprise », European Small Business Seminar, Gand-Bruxelles, 21 p.

Peterson, R. et N.R. SMITH (1986), «Entrepreneurship : a culturally appropriate combination of craft and opportunity », dans Frontiers of Entrepreneurship Research, Babson College, p. 1-11.

RAMus, V. (1989), "A longitidunal study of new business projects : first results about the early development of new ventures ", dans P. DuBIN (éd.), The Evaluation of Entrepreneurial Projects and Profiles, Milan, EGEA, p. 73-92.

RAMUS, V. et Y.-F. LIVIAN, « Relations between types of risks and new ventures success », dans S. BIRLEY (éd.), Building European Ventures, Amsterdam, Elsevier science publishers B.V., p. 198-213.

Rigaud, G. (1987), "Le démarrage d'activités nouvelles: typologie des risques et financement des projets », Banque, 504, p. 244-260.

SANDBERG, W.R. et C.W. HOFER (1987), «Improving new venture performance : the role of strategy, industry structure, and the entrepreneur ", Journal of Business Venturing, vol. 2, $\mathrm{n}^{\circ} 1$, p. 5-28.

SeXton, D.L. et N.B. Bowman (1986), «Validation of a personality index : comparative psychological characteritics analysis of female entrepreneurs, managers, entrepreneuship students and business students ", dans Frontiers of Entrepreneurship Research, Babson College, p. 40-51.

SHAILER, G. (1989), «The predictability of small entreprise failures : evidence and issues », International Small Business Journal, vol. 7, $n^{\circ} 4$, p. 54-58.

SMallboNe, D. (1990), "Success and failure in new business start-ups », International Small Business Journal, vol. 8, $\mathrm{n}^{\circ} 2$, p. 34-47.

SMITH, N.R., J.S. BRACKER et J.B. MINER (1987), "Correlates of firm and entrepreneur success in technologically innovative companies ", dans Frontiers of Entrepreneurship Research, Babson College, p. 337-353.

STEINER, M.P. et O. SolEM (1988), «Factors for success in small manufacturing firms », Journal of Small Business Management, vol. 26, $\mathrm{n}^{\circ} 1$, p. 51-56. 
STUART, R. et P. ABETTI (1987), «Start up ventures : towards the prediction of initial success ", Journal of Business Venturing, vol. 2, $n^{\circ} 3$, p. 215-230.

STUART, R. et P. ABETTI (1988), « Field study of technical ventures - Part III : the impact of entrepreneurial and management experience on early performance », dans Frontiers of Entrepreneurship Research, Babson College, p. 177-193.

VAN DE Ven, A., R. Huston et D.M. Schroeder (1984), « Designing new business startups », Journal of Management, vol. $10, n^{\circ} 1$.

VIENNET, H. (1990), « Survivre : premier souci des jeunes entreprises », INSEE Première, 110 , novembre $1990,4 \mathrm{p}$.

WARD, R. et R. RANDALL (1989), "Competitive advantage in the new business venture : the role of social networks », dans P. Dubini (éd.), The Evaluation of Entrepreneurial Projects and Profiles, Milan, EGEA, p. 43-60.

Woo, C.Y., A. CoOper et W. Dunkelberg (1988), «Entrepreneurial typologies : definitions and implications ", dans Frontiers of Entrepreneurship Research, Babson College, p. 165-176. 